\title{
CellSonic Rules of Medicine: Safety, Lowest Cost, Quickest Cure, Easy To Use, Support for All
}

\author{
Andrew Hague* \\ *President, professor of Advanced Medicine, CellSonic: Manufacturers of Medical Equipment, United Kingdom
}

Received: September 14, 2017; Accepted: September 22, 2017; Published: October 16, 2017

*Corresponding author: Andrew Hague, President, Professor of Advanced Medicine, CellSonic: Manufacturers of Medical Equipment, United Kingdom, Tel: +1315210 6307; E-mail: cellsonic.beauty@gmail.com

\section{Safety}

There must be:

No side effects.

No risk to the doctors and nurses.

No risk to the environment.

CellSonic VIPP meets all three requirements for all its applications from wounds to cancer. Contrast this with drugs; all have side effects and disposal can cause contamination. Antibacterial resistance is rising. Use CellSonic VIPP to kill infection instead of drugs.

If an inexperienced CellSonic VIPP operator aims at healthy cells in the body instead of the target site, there is no harm done. If a patient is given the wrong dose of drugs it could be fatal.

\section{Lowest Cost}

The only cost that matters is the cost of cure and in every application with CellSonic VIPP it has the lowest cost. This is achieved by involving a doctor or nurse less than other methods. The patient is less disrupted and the hospital used less.

\section{Quickest Cure}

CellSonic VIPP has the quickest cure and often the only cure. In many cases, CellSonic VIPP is the only cure for cancer and succeeds in two to three weeks. To cause the islets on the pancreas to generate insulin can take three months and is still the only cure for diabetes. Non-healing wounds are healed with CellSonic VIPP. Large wounds that have been open for years are made to heal in a few weeks to a few months. CellSonic VIPP is the only cure for gangrene.

\section{Easy to Use}

It takes five minutes to learn how to use the CellSonic VIPP machine. The operator can be shown where to aim the shock head and the programming of the machine stops it when the required number of pulses has been delivered. The wide beam of the pulse makes it easy to aim at the target. If pulses are off target, they do no harm.

\section{Support and Training}

Distributors receive training. They teach their customers and in turn experienced users teach newcomers. Everyone knows everyone else so that CellSonic is more of a family than a company. [Figure: 1,2]

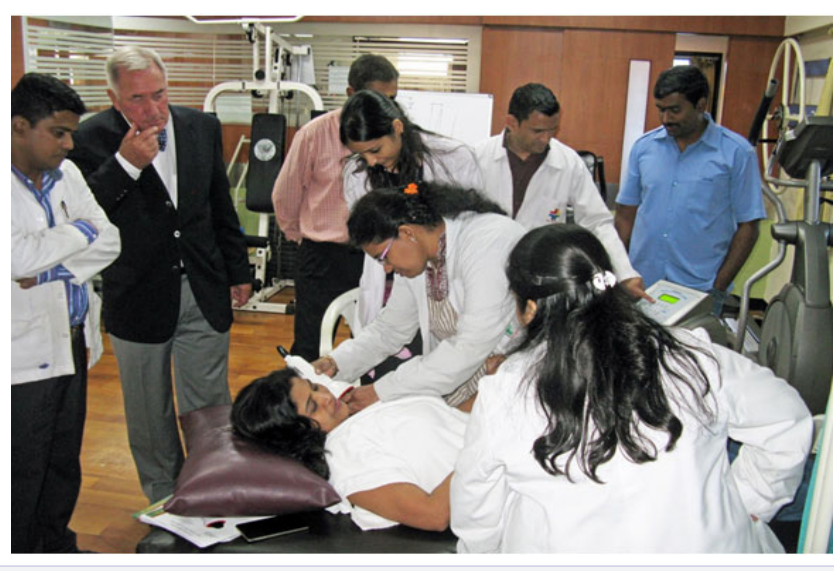

Figure 1

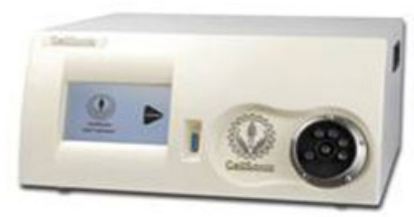

Figure 2 